\title{
BACKWARD METHOD OF CHARACTERISTICS IN RADIATIVE HEAT TRANSFER
}

\author{
Kamal M. Katika and Laurent Pilon \\ Mechanical and Aerospace Engineering Department \\ Henri Samueli School of Engineering and Applied Science \\ University of California, Los Angeles - Los Angeles, CA 90095, USA \\ E-mail: pilon@seas.ucla.edu
}

\begin{abstract}
This paper presents the backward method of characteristics for simulating multidimensional transient and steady state radiative heat transfer. This method is based on the method of characteristics that follows photons along their pathlines. It makes use of a fixed grid and unlike the conventional method of characteristics, it follows the photons backward in time. The method (1) is accurate, (2) unconditionally stable, (3) can be used for solving coupled equations using other numerical schemes. Numerical results obtained for various cases solved were in good agreement with analytical and numerical solutions reported in literature. The method is particularly appropriate for simulating multidimensional, transient radiative transport, and/or coupled heat transfer problems.
\end{abstract}

\section{NOMENCLATURE}

$\begin{array}{ll}I_{b} & \text { blackbody radiation intensity } \\ N & \text { conduction to radiation parameter } \\ N_{x} & \text { number of nodes in x-direction } \\ N_{y} & \text { number of nodes in y-direction } \\ N_{z} & \text { number of nodes in z-direction } \\ N_{\theta} & \text { number of discrete polar angles per octant } \\ N_{\phi} & \text { number of discrete azimuthal angles per octant } \\ \Delta t & \text { time step } \\ \hat{\mathbf{s}} & \text { direction unit vector } \\ \Delta z & \text { element size along z axis }\end{array}$

\section{Greek symbols}

$\begin{array}{ll}\mu & \text { direction cosine }(=\cos \theta) \\ \Psi & \text { dimensionless emissive power }=\left(T^{4}-T_{1}^{4}\right) /\left(T_{2}^{4}-T_{1}^{4}\right)\end{array}$

\section{Subscripts}

$\begin{array}{ll}1,2 & \text { at location, " } 1 \text { " or " } 2 \text { " } \\ b & \text { refers to blackbody behavior } \\ L & \text { value at location } \mathrm{z}=\mathrm{L} \\ \lambda & \text { at a given wavelength, or per unit wavelength }\end{array}$




\section{INTRODUCTION}

Radiation transport in participating media is important in laser materials processing, remote sensing, and many other applications. Of late, with the advent of devices like short pulse lasers and their applications ranging from micromachining to optical tomography, the study of transient radiative transfer has come into prominence [1]. Lasers have also been used to destroy tumors in the human body by heating them up while sparing the surrounding tissue [2]. Since the temperature distribution depends on the irradiation and optical properties of the tissue, it is important to study the transport of radiation in tissue and other such media. Radiative heat transfer is also important in industrial furnaces, combustion chambers, re-entry applications where combined modes of heat transfer are involved and radiation is the dominant mode.

The governing equation for radiative heat transfer is the radiative transfer equation (RTE). It can be written as [3],

$$
\frac{1}{c} \frac{\partial I_{\lambda}}{\partial t}+(\hat{\mathbf{s}} \cdot \nabla) I_{\lambda}=\kappa_{\lambda} I_{b \lambda}-\kappa_{\lambda} I_{\lambda}-\sigma_{s \lambda} I_{\lambda}+\frac{\sigma_{s \lambda}}{4 \pi} \int_{4 \pi} I_{\lambda}\left(\hat{\mathbf{s}}_{i}\right) \Phi_{\lambda}\left(\hat{\mathbf{s}}_{i}, \hat{\mathbf{s}}\right) \mathrm{d} \Omega_{i}
$$

where all the symbols have their usual meanings and $c$ is the speed of light in the medium.

The most widely used methods to solve the RTE are, among others, the discrete ordinates method, the Monte Carlo method, and the finite volume method. The discrete ordinate method has several disadvantages: (1) the so called "ray-effect" and (2) the difficulty in dealing with specular boundaries. The ray effect occurs when the continuous incident intensity distribution is approximated by a discrete angular quadrature in the numerical evaluation of the incident flux integral causing an oscillatory behavior in the boundary heat flux. At specular boundaries, the reflected or transmitted beams might not coincide with the discrete ordinates and are thus difficult to deal with.

The Monte Carlo method involves tracing the history of a statistically meaningful random sample of photons from their points of emission to their points of absorption or to the point they exit the media. The advantage of the Monte Carlo method is that even the most complicated problem can be solved with relative ease in formulation. The method is suited for problems where the complexity of formulation and the solution effort involved are too great for conventional means. The disadvantage of this method is that, it is subject to statistical error [3]. It is also computationally time consuming and also demands a lot of computer memory [4]. Finally, it is difficult to couple with CFD methods for transport problems involving other modes of heat transfer or fluid flow.

Finite volume methods have been developed by Chui and Raithby to solve the RTE [5]. The procedure involves discretizing the angular domain into a finite number of control angles over which radiant energy is conserved. Chai et al. [6] also used a similar approach to solve the RTE in irregular geometries. Murthy and Mathur [7] extended the method to unstructured meshes to compute radiative heat transfer in absorbing, emitting and scattering media. Finite volume techniques, though very popular, are susceptible to false scattering which depends on the spatial discretization scheme used. This might lead to incorrect results especially in multidimensional transient radiative transfer [8].

Rukolaine et al. [9] have discussed the method of characteristics as a technique to solve the radiative transport equation for axisymmetric domains with both diffuse and specularly reflecting boundaries. They reported numerical solutions to various problems involving cylindrical geometries, absorbing and emitting media and various boundary conditions such as black top and bottom walls at constant temperature. They also applied it to the simulation of combined 
radiative and conductive heat transfer in the growth of semitransparent crystals from a melt. In spite of good accuracies reported, the method can only be used for axi-symmetric cases like cylinders and cannot simulate transient phenomena.

The backward or reverse Monte Carlo has been developed as an alternative approach when solutions are needed at particular locations and times [10]. The method is similar to the traditional Monte Carlo method, except that the photons are tracked in a time-reversal manner. The photon bundles are traced from the detector to the source rather than from the source to the detector as in the conventional Monte Carlo method. There is no need to keep track of photons which do not reach the detector and so the RMC is much faster than the traditional Monte Carlo method. The Reverse Monte Carlo method was successfully applied by Hsu et al. [11] for 1-D transient radiative transfer in participating media. But again, it has the same disadvantages as the Monte Carlo method in terms of statistical accuracy.

This paper aims at presenting the backward method of characteristics as a means of solving the transient and steady-state radiative transfer equation as well as combined heat transfer and fluid flow problems. The new method addresses all the above mentioned issues and shortcomings of existing methods. First, a brief description of the method is given followed by simulations of test cases for which exact and numerical solutions have been reported in the literature.

\section{BACKWARD METHOD OF CHARACTERISTICS}

The method of characteristics consists of transforming the radiative transport equation, a partial integro-differential equation into an ordinary integro-differential equation along the photon pathlines. The conventional method of characteristics identifies particles at initial time $t=t_{0}$ and follows them along the characteristic at subsequent times. Though the direct method results in very accurate solutions, it has several disadvantages. Step sizes along different characteristic curves may be different and so the solution maybe obtained at different times on each characteristic curve. Also, the characteristic curves may coalesce or spread apart due to non-uniform velocities in non-linear problems resulting in a highly non-uniform grid [12]. The backward method of characteristics on the other hand, follows particles backward in time and uses a pre-specified grid in the region of interest. Thus the solution is obtained at the same values of time on all characteristic curves and overcomes the problems related to grid deformation [12]. Consider a Cartesian coordinate system. The characteristic curve in physical space is defined as

$$
\frac{\mathrm{d} x}{\mathrm{~d} t}=c \sin \theta \cos \phi, \quad \frac{\mathrm{d} y}{\mathrm{~d} t}=c \sin \theta \sin \phi, \text { and } \quad \frac{\mathrm{d} z}{\mathrm{~d} t}=c \cos \theta
$$

Then, the total derivative $\mathrm{D} I_{\lambda} / \mathrm{D} t$ along this characteristic curve can be written as

$$
\frac{\mathrm{D} I_{\lambda}}{\mathrm{D} t}=\frac{\partial I_{\lambda}}{\partial t}+\frac{\mathrm{d} x}{\mathrm{~d} t} \frac{\partial I_{\lambda}}{\partial x}+\frac{\mathrm{d} y}{\mathrm{~d} t} \frac{\partial I_{\lambda}}{\partial y}+\frac{\mathrm{d} z}{\mathrm{~d} t} \frac{\partial I_{\lambda}}{\partial z}
$$

The RTE along the characteristic curves in $(x, y, z, t)$ space simplifies to

$$
\frac{1}{c} \frac{\mathrm{D} I_{\lambda}}{\mathrm{D} t}=\kappa_{\lambda} I_{b \lambda}-\kappa_{\lambda} I_{\lambda}-\sigma_{s \lambda} I_{\lambda}+\frac{\sigma_{s \lambda}}{4 \pi} \int_{4 \pi} I_{\lambda}\left(\hat{\mathbf{s}}_{i}\right) \Phi_{\lambda}\left(\hat{\mathbf{s}}_{i}, \hat{\mathbf{s}}\right) \mathrm{d} \Omega_{i}
$$

Thus, a partial differential equation is converted to an ordinary integro-differential equation. To solve Equation (4), the radiation intensities and temperatures are initialized at all points in the computational domain. Figure 1 shows a 3-D computational cell in Cartesian coordinates.

For a given polar angle $\theta_{n}$, an azimuthal angle $\phi_{l}$ and for all internal grid points $\left(x_{a}, y_{b}, z_{c}\right)$ where photons are located at time $t+\Delta t$, the photon's position at time $\mathrm{t}$ is calculated as

$$
x_{n}=x_{a}-c \sin \theta_{n} \cos \phi_{l} \Delta t, \quad y_{n}=y_{b}-c \sin \theta_{n} \sin \phi_{l} \Delta t, \text { and } \quad z_{n}=z_{c}-c \cos \theta_{n} \Delta t
$$


The values of the variables $I_{\lambda}$ at $\left(x_{n}, y_{n}, z_{n}\right)$ and time $t$ are obtained by Lagrangian interpolation

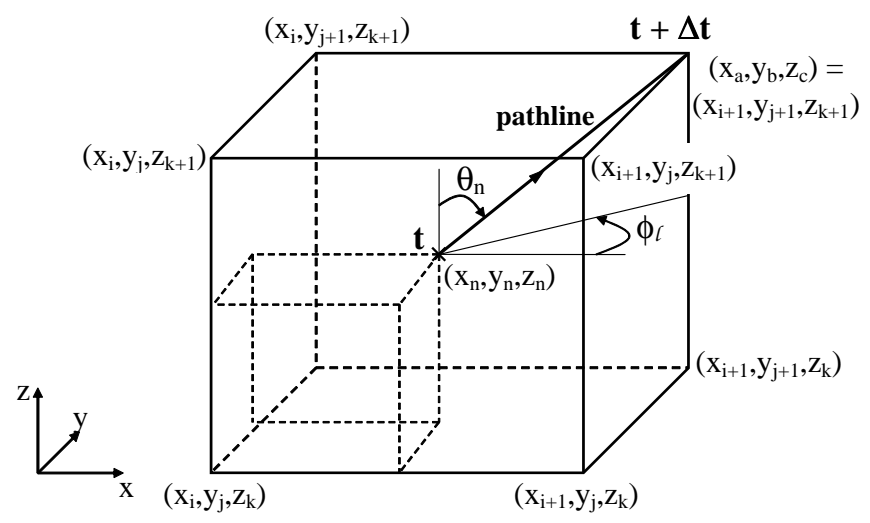

Fig. 1: Typical computational cell used for inverse marching method containing the pathline of the photons.

using their values at time $t$ at the eight corners of the computational cell in which $\left(x_{n}, y_{n}, z_{n}\right)$ is located. Then, Equation (4) is solved by the fourth order Runge-Kutta method at location $\left(x_{a}, y_{b}, z_{c}\right)$ at time $t+\Delta t$. The integral on the right hand side of Equation (4) is estimated by the 3/8 Simpson numerical integration method. Then the boundary conditions are imposed. The calculations are repeated for all the discretized values of polar angles and azimuthal angles. The backward method of characteristics has the following main advantages :

- Unlike finite volume techniques which propagate the information along the coordinate axis, the backward method of characteristics propagates information along the photon pathlines. Like the Monte Carlo method, it respects the physics of radiative transport resulting in extremely accurate numerical results.

- Since the method uses a pre-specified computational grid, it can be easily coupled with other numerical techniques such as finite volume or finite difference schemes.

- It can be used for both transient and steady state analysis with great accuracy and without problems of numerical instability.

- There is no false scattering unlike the finite volume method.

There are also a few disadvantages in using the backward method of characteristics to solve the radiative transport equation. The backward projected characteristic curves do not intersect the known solution surface at the pre-specified grid points and so the initial data at the backward projected characteristics must be determined by interpolation. It is also computationally expensive for steady state problems compared to traditional methods like finite volume and discrete ordinate methods (DOM) as it solves a steady state problem as a limiting case of a transient problem.

\section{RESULTS AND DISCUSSION}

For validation purposes, the numerical results obtained with the backward method of characteristics for a set of test cases have been compared with analytical solutions or numerical results reported in literature and using different numerical schemes. For the sake of clarity, spectral dependencies were not considered in these cases but could have been included without any modifications in the RTE or the associated computer code. 


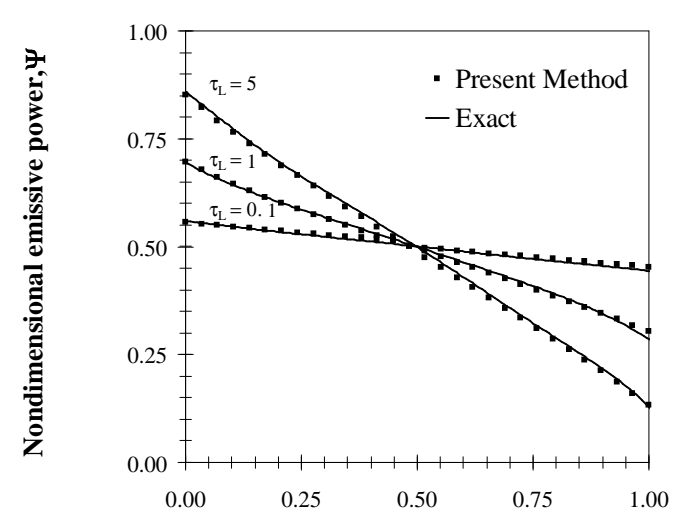

Nondimensional location, $\tau / \tau_{\mathrm{L}}=\mathrm{z} / \mathrm{L}$

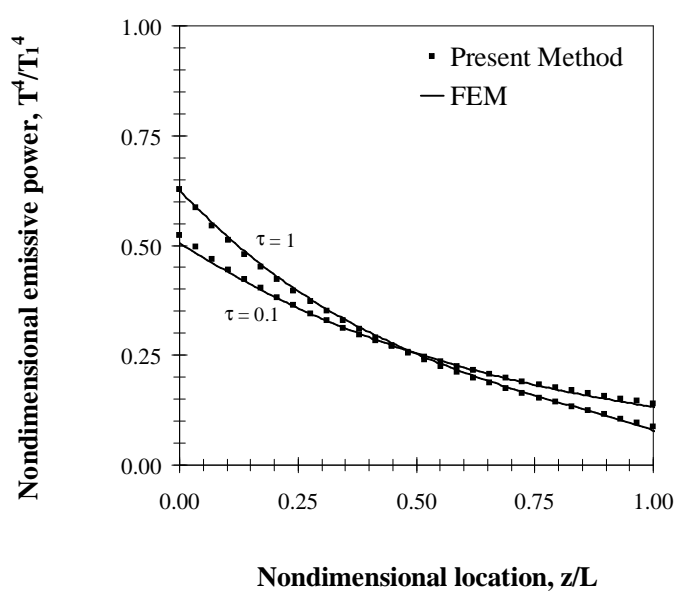

Fig. 3: Nondimensional temperature distribution for a 2-D rectangular enclosure with gray participating media at radiative equilibrium.

\section{Radiative equilibrium of an anisotropically scattering medium}

The problem of radiative equilibrium in a one-dimensional, plane parallel, absorbing, emitting and anisotropically scattering gray medium has been solved analytically by Modest and Azad [13]. The computational domain was discretized into a $N_{x} \times N_{y} \times N_{z}$ grid where $N_{x}, N_{y}$ and $N_{z}$ represent the number of grid points in the $\mathrm{x}-, \mathrm{y}-$, and $\mathrm{z}$ - directions, respectively. The angular space was discretized into $N_{\theta} \times N_{\phi}$ directions over one octant, i.e. the range of $\theta$ from 0 to $\pi / 2$ was divided into $N_{\theta}$ directions and $\phi$ varying from 0 to $\pi / 2$ was divided into $N_{\phi}$ directions. The integral on the right-hand-side of Equation (1) corresponding to the in-scattering term was computed numerically using an unstructured 3/8 Simpson's scheme and discretizing the angular directions [12]. A discretization of 30 grid points along the $z$-direction and an angular discretization of $8 \times 1$ directions per octant was used. Figure 2 shows the solution computed by Modest and Azad [13] using a linear phase function and the results obtained with the present numerical method. The mean error in temperature was less than $1.5 \%$ for $\tau_{L}=0.1$ and $\tau_{L}=1.0$ while it was $2.5 \%$ for $\tau_{L}=1.0$. The maximum error was less than $3 \%$ for $\tau_{L}=0.1$ and $\tau_{L}=1.0$ while it went up to $7 \%$ at the boundary for $\tau_{L}=5.0$. The error increased for increasing values of optical thickness and was maximum at the boundaries. The error can be further reduced by increasing the number of $z$-direction gridpoints and using a finer angular discretization.

\section{Radiative heat transfer in a 2-D rectangular enclosure with gray participating media}

Razzaque et al. [14] have reported a solution of radiative heat transfer in a two-dimensional rectangular enclosure with gray participating media using a finite element technique. The surfaces of the enclosure were assumed to be black. The lower surface of the enclosure was assumed to be at temperature $T_{1}$ while the remaining three surfaces were maintained at 0 $\mathrm{K}$. The backward method of characteristics was employed to solve the radiative transport equation in a 2-D rectangular enclosure with gray participating media. A spatial discretization of $30 \times 8 \times 30$ and an angular discretization of $12 \times 12$ directions per octant was used. Figure 3 compares the results obtained with the present method with those reported by Razzaque et al. [14] for optical thicknesses of $\tau=0.1$ and $\tau=1$. The error increased for increasing values of optical thickness. The mean error was less than $1.5 \%$ for all the optical thicknesses while the maximum error of $9 \%$ occurred at the boundary for $\tau=1$. The maximum error was $7 \%$ by 
making use of a grid with 60 grid points in the $x$ and $z$ directions and $12 \times 12$ directions per octant. Further improvements can be made by using a finer discretization on $\theta$.

\section{Transient radiative transfer in a scattering, absorbing, and emitting slab}

The transient effect of radiation is important in short pulse laser transport where the input pulse temporal width is usually of the order of pico and femto seconds similar to the order of the radiation propagation time in the system. Consider the case of a non-emitting absorbing and isotropically scattering medium subjected to a transient unit step function diffuse emissive power on one side and the other side being cold with optical thickness $\tau=1$ and scattering albedo $\omega=0.5$. Initially, the medium is assumed to be at a temperature of $0 \mathrm{~K}$ and intensities everywhere in the medium to be zero. Then, at $t=0$ a diffuse intensity $I(z=0, \mu>0, t)=$ 1 is imposed. A spatial discretization of 101 points along the $z$-direction and an angular discretization of $12 \times 1$ directions per octant is used. The time interval $\Delta t$ is equal to $\Delta z / c$ where $\Delta z=L / 100$. Figure 4 compares the radiative flux distribution as a function of time obtained with the backward method of characteristics and the exact solution reported by Hsu and Tan [15]. The error in the radiative flux computed by the present method and that reported by Hsu and Tan was less than $1 \%$ for all values of $t / \Delta t$ except at the radiation front where it went up to $4 \%$ for $t / \Delta t=30$ and $t / \Delta t=60$ and $8 \%$ at a single point for $t / \Delta t=90$. The maximum errors occurred at the radiation front in all cases. This error can be reduced by using finer discretizations for $\theta$ and $z$. The same problem was solved by Chai [16] using the finite volume method. Though he was able to predict the radiative flux correctly once the radiation reached the opposite side, he was not able to exactly capture the wavefront at earlier times. Finite volume techniques are susceptible to false scattering and this depends on the spatial discretization scheme used. As a result of false scattering, a smearing of the wavefront is observed [8].

\section{Steady state of a slab with combined conduction and radiation}

Combined radiation and conduction is very important in applications such as thermal insulations and materials processing. Consider the case of steady-state heat transfer through a one-dimensional absorbing-emitting, non-scattering gray medium confined between two parallel, black, isothermal, plates. Assuming that the thermal conductivity $k$ is uniform throughout the slab and in the absence of heat generation, the equation for combined radiation and conduction can be written as

$$
k \frac{\mathrm{d}^{2} T}{\mathrm{~d} z^{2}}=\kappa\left(4 \pi I_{b}-G\right)
$$

where, $I_{b}=\sigma T^{4} / \pi$ and the incident radiation is denoted by $G$ and is expressed as $\int_{-1}^{1} I \mathrm{~d} \mu$. The boundary conditions are $T(z=0)=T_{1}$, and $T(z=L)=T_{2}=0.5 T_{1}$. The dimensionless number $N=k \kappa / 4 \sigma T_{1}^{3}$ is defined to determine the relative importance of conductive and radiative heat fluxes for optically thick slabs [3]. Now, a central difference scheme is employed to express the second order derivative of the temperature in the energy equation resulting in a tridiagonal system of equations. To solve these equations, an initial temperature profile is guessed and the radiative transfer equation is solved to compute the intensities at steady state. The right hand side of Equation (6) is then computed. A new temperature profile is determined by inverting the tridiagonal matrix for temperature. This procedure is repeated till a converged solution is obtained. An underrelaxation parameter of 0.2 was used in cases when radiation was the dominant mode of heat transfer. It should be noted here that the same grid used for solving the RTE is made use of in solving Equation (6). A spatial discretization of 30 along the $z$-direction and an angular discretization of $24 \times 1$ directions per octant was used. Figure 5 shows the vari- 


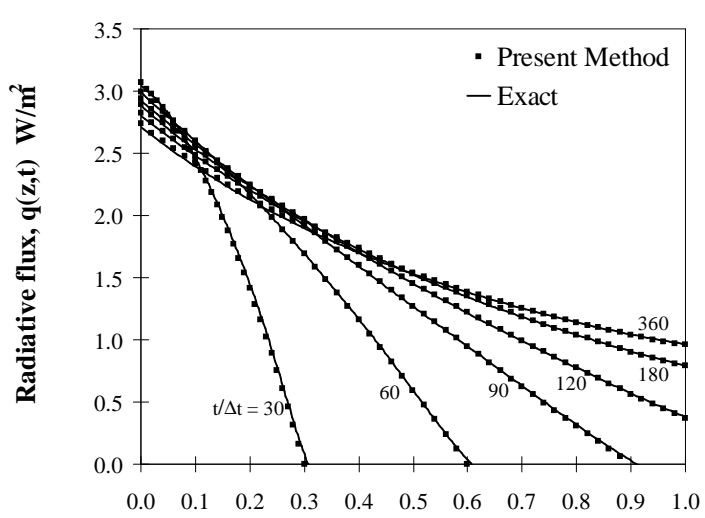

Nondimensional location, $\mathbf{z} / \mathbf{L}$

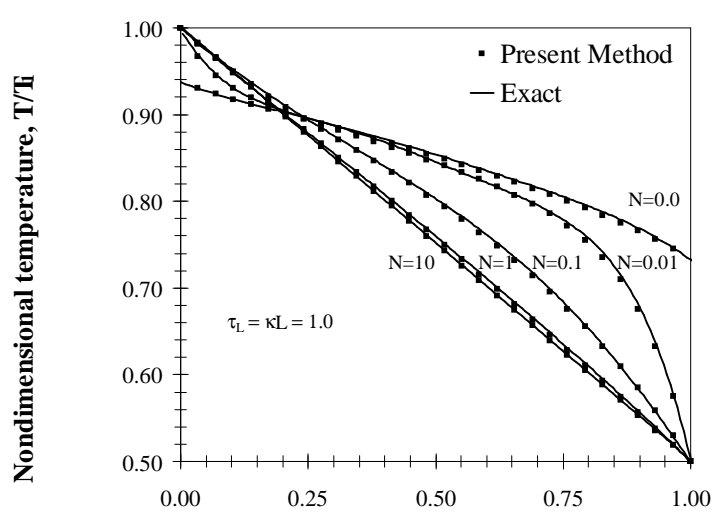

Nondimensional position, $\mathbf{z} / \mathbf{L}$
Fig. 4: radiative flux distribution at different times in a homogeneous medium with one diffusely emitting boundary.
Fig. 5: Nondimensional temperature distribution for combined radiation and conduction across a gray slab bounded by black plates.

ation in non-dimensional temperature $T / T_{1}$ within the slab for an optical thickness of $\tau_{L}=1$ and various values of $\mathrm{N}$ calculated using the present method and the exact solution reported by Viskanta and Grosh [17]. The error in the values of temperature computed by the present method and the solution reported by Viskanta et al. [17] was less than $0.5 \%$ for all values of $\mathrm{N}$ between 10 and 0 . Radiative heat fluxes at the hot wall were computed for a representative set of optical thicknesses and $N$ values and they were found to be within $5 \%$ reported by Viskanta and Grosh [17]. Again, the error increased with increasing values of optical thickness.

\section{CONCLUSION}

The results described above confirm the numerical scheme and its capability in handling various problems encountered in common engineering practice. The computer program used to implement the described method was not optimized. Instead, the study has been aimed at demonstrating the applicability of the method to various problems encountered in radiative heat transfer. Various improvements can be made to decrease the computational time :

- The number of discrete directions can be reduced or replaced by quadrature as commonly used in the discrete ordinate method to accelerate the computation of the in-scattering term.

- Another point to be noted is that the 1-D and 2-D cases described were solved as special cases of a general 3-D problem thus increasing computational times. These could have been reduced by simply solving the RTE in 1-D or 2-D.

- Though the case of a diffuse boundary condition was not discussed here, it can be easily implemented as done by Rukolaine et al. [9]. Also, the case of of specularly reflecting boundaries using the backward method of characteristics has been recently described for phonon transport by Pilon et al. [18]. A similar treatment can be applied to partially reflecting and transmitting boundaries.

- Apart from the various cases described above, (i) the case of combined convection and radiation and (ii) multiphase laminar or turbulent flames can also be solved using this method with very minor modifications. 
- Also since the method is fully explicit it can be easily adapted for parallel computing. This could be a useful feature in real time analysis and real time inverse analysis for biomedical diagnostics or other remote sensing applications.

The backward method of characteristics has been presented as a way to solve the radiative transport equation. It has been shown that the method can handle various problems including multidimensional, transient and steady-state thermal radiation transfer problems. The accuracy of the method demonstrated in computing the transient radiative heat flux puts it at an advantage over conventional techniques like finite volume which are computationally expensive and susceptible to various inaccuracies such as smearing of energy distribution observed due to false scattering [8]. An additional advantage lies in the fact that this method makes use of a fixed grid. Thus, it can be coupled easily with other methods. This can be a very valuable feature in problems involving radiative heat transfer in reactive flows where the velocity, temperature, concentration and the intensity fields have to be solved. This is a big advantage of this method over Monte Carlo techniques where individual photons are tracked to solve the RTE and so there is no fixed grid and as a result are hard to couple with finite volume techniques.

\section{REFERENCES}

1. S. Kumar and K. Mitra, "Microscale aspects of thermal radiation transport and laser applications", Advances in Heat Transfer, vol. 33, pp. 187-294, 1999.

2. C-H. Huang and C-Y. Huang, "An inverse biotechnology problem for estimating the optical diffusion and absorption coefficients of tissue", International Journal of Heat and Mass Transfer, vol. 47, pp. 447-457, 2004.

3. M. F. Modest, Radiative Heat Transfer, McGraw-Hill, New York, NY, 1993.

4. Z. Guo, S. Kumar, and K.-C. San, "Multidimensional Monte Carlo simulation of shortpulse transport in scattering media", Journal of Thermophysics and Heat Transfer, vol. 14, no. 4, pp. 504-511, 2000.

5. G. D. Raithby and E. H. Chui, "A finite-volume method for predicting a radiant heat transfer in enclosures with participating media", Journal of Heat Transfer, vol. 112, pp. 415-423, 1990.

6. J. Chai, G. Parthasarathy, S. Patankar, and H. Lee, "A finite-volume radiation heat transfer procedure for irregular geometries", Journal of Thermophysics and Heat Transfer, vol. 9, no. 3, pp. 410-415, 1994.

7. J.Y. Murthy and S.R. Mathur, "A finite volume method for radiative heat transfer using unstructured meshes", Journal of Thermophysics and Heat Transfer, vol. 12, pp. 313-321, 1998.

8. J. C. Chai, P-F. Hsu, and Y. C. Lam, "Three-dimensional transient radiative transfer modeling using the finite-volume method", Journal of Quantitative Spectroscopy and Radiative Transfer, In Press, 2003.

9. S. A. Rukolaine, M . G. Vasilyev, V. S. Yuferev, and A. O. Galyukov, "Numerical solution of axisymmetric radiative heat transfer problems in arbitrary domains using the characteristic method", Journal of Quantitative Spectroscopic and Radiative Transfer, vol. 73, pp. 205-217, 2002. 
10. M. F. Modest, "Backward Monte Carlo simulations in radiative heat transfer", ASME Journal of Heat Transfer, vol. 125, pp. 57-62, 2003.

11. X. Lu and P.-F. Hsu, "Reverse Monte Carlo method for transient radiative transfer in participating media", Proceedings of the ASME International Mechanical Engineering Congress and Exposition, 2003.

12. J. D. Hoffman, Numerical Methods for Engineers and Scientists, McGraw Hill, New York, NY, 1998.

13. M. F. Modest and F. H. Azad, "The influence and treatment of Mie-anisotropic scattering in radiative heat transfer", ASME Journal of Heat Transfer, vol. 102, pp. 92-98, 1980.

14. M. M. Razzaque, D. E. Klein, and J. R. Howell, "Finite element solution of radiative heat transfer in a two-dimensional rectangular enclosure with gray participating media", ASME Journal of Heat Transfer, vol. 105, pp. 933-936, 1983.

15. Z-M Tan and P-F. Hsu, "An integral formulation of transient radiative transfer", ASME Journal of Heat Transfer, vol. 123, pp. 466-475, 2001.

16. J.C. Chai, "One-dimensional transient radiation heat transfer modeling using a finitevolume method", Numerical Heat Transfer, Part B, vol. 44, pp. 187-208, 2003.

17. R. Viskanta and R.J.Grosh, "Heat transfer by simultaneous conduction and radiation in an absorbing medium", Journal of Heat Transfer, vol. 84, pp. 63-72, 1962.

18. L. Pilon and K. Katika, "Backward method of characteristics for simulating microscale energy transport", to appear in ASME Journal of Heat Transfer, vol. 126, No.4, 2004. 